Full length article

\title{
A free energy landscape perspective on the nature of collective diffusion in amorphous solids
}

\author{
Yun-Jiang Wang a, b, *, Jun-Ping Du ${ }^{\text {c, d }}$, Shuhei Shinzato ${ }^{\text {, }}$, Lan-Hong Dai a, b, **, \\ Shigenobu Ogata ${ }^{\text {c, d, } * * *}$ \\ a State Key Laboratory of Nonlinear Mechanics, Institute of Mechanics, Chinese Academy of Sciences, Beijing, 100190, China \\ ${ }^{\mathrm{b}}$ School of Engineering Science, University of Chinese Academy of Sciences, Beijing, 100049, China \\ ${ }^{c}$ Department of Mechanical Science and Bioengineering, Graduate School of Engineering Science, Osaka University, Osaka, 560-8531, Japan \\ ${ }^{\mathrm{d}}$ Center for Elements Strategy Initiative for Structural Materials (ESISM), Kyoto University, Sakyo, Kyoto, 606-8501, Japan
}

\section{A R T I C L E I N F O}

\section{Article history:}

Received 3 April 2018

Received in revised form

23 June 2018

Accepted 11 July 2018

Available online 17 July 2018

\section{Keywords:}

Metallic glasses

Collective diffusion

Accelerated molecular dynamics

Free energy landscape

\begin{abstract}
A B S T R A C T
The nature of collective diffusion in amorphous solids is in strong contrast with diffusion in crystals. However, the atomic-scale mechanism and kinetics of such collective diffusion remains elusive. Here the free energy landscape of collective diffusion triggered by single atom hopping in a prototypical $\mathrm{Cu}_{50} \mathrm{Zr}_{50}$ metallic glass is explored with well-tempered metadynamics which significantly expands the observation timescale of diffusion at atomic-scale. We clarify an experimentally suggested collective atomic diffusion mechanism in the deep glassy state. The collective nature is strongly temperature-dependent. It evolves from string-like motion with only several atoms to be large size collective diffusion at high temperature, which can promote the atomic transport upon glass transition temperature. We also clarify the apparent diffusivity is dominated by the highest free energy barrier of atomic diffusion among widely distributed free energy barriers due to the dynamic heterogeneity of metallic glass, which suggests the sequential nature of diffusion is a proper assumption to the metallic glasses with dynamic heterogeneity. The temperature and pressure dependence of diffusion free energy landscape are further quantified with activation entropy, $(19.6 \pm 2.5) k_{\mathrm{B}}$, and activation volume, $(7.9 \pm 3.4) \AA^{3}$, which agree quantitatively with experiments. Laboratory timescale simulations of atomic diffusion brings physical insights into the unique atomic motion mechanism in non-crystalline materials.
\end{abstract}

๑) 2018 Acta Materialia Inc. Published by Elsevier Ltd. All rights reserved.

\section{Introduction}

Metallic glasses (MGs) are thermodynamically metastable structures which are prone to undergo various atomic rearrangement processes that affect their macroscopic behaviors. The longrange atomic diffusion is such a fundamental process in glass and its melts and continues to be a focus of materials science and condensed matter physics [1-8]. A microscopic understanding of diffusion is of vital importance to various critical phenomena of

\footnotetext{
* Corresponding author. State Key Laboratory of Nonlinear Mechanics, Institute of Mechanics, Chinese Academy of Sciences, Beijing, 100190, China.

** Corresponding author. State Key Laboratory of Nonlinear Mechanics, Institute of Mechanics, Chinese Academy of Sciences, Beijing 100190, China.

*** Corresponding author. Department of Mechanical Science and Bioengineering Graduate School of Engineering Science, Osaka University, Osaka, 560-8531, Japan. E-mail addresses: yjwang@imech.ac.cn (Y.-J. Wang), lhdai@lnm.imech.ac.cn (L.-H. Dai), ogata@me.es.osaka-u.ac.jp (S. Ogata).
}

MGs, such as structural relaxation [9-12] and rejuvenation [13-15], crystallization [4,16], glass transition [1,5-7,17-20], and viscoelastic/plastic deformation [21-23], all of which are both of fundamental and broad scientific curiosity, and of technical importance towards applications. Unlike crystals, the diffusion mechanism in amorphous solids has not been thoroughly understood since it is hard to envisage an on-site hopping of single atoms or any other 'point defect' in the disordered structure [2,3]. Various experiments suggested that the collective diffusion involving multiple atoms plays a critical role in the long-range atomic transport [1,24,25]. Recently, advancement has been achieved to study in-situ diffusion in both crystalline [26] and amorphous alloys [27-29] by using X-ray photon correlation spectroscopy. Strong correlation has been established between the heterogeneous dynamics and cooperative atomic motion in amorphous alloys $[27,28,30]$. However, an in-situ atomic-level observation of such processes still remains extremely challenging in either experiments [26] or computer simulations. 
Molecular dynamics (MD) has made great success in simulating atomic diffusion in MGs and their melts, providing important microscopic insights into the diffusion processes [12,31-33]. For example, MD has recognized a collective chain-type (or string-like) cooperative motion in MG at elevated temperature in the vicinity of glass transition or above [3,12,32,33], the fine details of the stringlike excitation and how such excitations evolve with temperature during very long temperature ramps [34-37]. MD also well substantiated the mode-coupling theory (MCT) in explaining the glass transition phenomenon as a dynamical phase transition [31]. However, diffusion is a rare event well below the glass transition temperature $T_{\mathrm{g}}$, which is far out of the classical MD time window $(\sim \mathrm{ns})$ [12]. For a general thermally activated plastic mechanism with a free energy barrier of the order of $30 k_{\mathrm{B}} T$ (about $0.78 \mathrm{eV}$ at ambient temperature, $k_{\mathrm{B}} T$ is thermal energy), it takes about $1 \mathrm{~s}$ to observe such an event. This experimentally relevant timescale is about 10 orders of magnitude longer than the atomistic computational capacity of a modern supercomputer. This is why MD simulations were usually carried out at high temperature or under extreme stress conditions, at which the hopping time of atom or the waiting time of a plastic event is relevant to the prototypical MD timescale window [38].

Direct atomic-scale simulations in deep glassy state is rarely reported, in which regime diffusion is still important for mechanical deformation and structural relaxation [10,11]. Therefore, an experimental timescale understanding of the diffusion thermodynamics and its mechanism, in particular in the glassy state at ambient temperature, is of great interest to the glass physics community but remains as an unresolved issue [38]. Until recently, a concept of metadynamics was introduced for exploring the free energy surface (FES) or free energy landscape of many dynamic processes [39]. The configurational state is forced to escape from a local energy minimum with adding bias potential to the original FES. Similar methods have been established and utilized to understand phenomena of creep [40,41], dislocation mobilities [42-44], and diffusion [45-47] in crystals. It is now just started to overcome the timescale limitation in simulating the structural relaxation [48], viscosity [49], crystallization [50], shear and creep deformation of disorder materials [9,51-53].

In this work, the well-tempered metadynamics is conducted to explore a single-atom-triggered diffusion mechanism of a prototypical model glass over wide temperature range that has never been achieved by the classical atomistic simulations. We find a temperature-dependent string-like cooperative motion of atoms in glassy state, while the size of collective motion increases significantly with increasing temperature. This observation perhaps explains the enhanced diffusivity beyond the Arrhenius law at temperature near glass transition. The kinetics of collective motion is quantified with experimentally comparable activation entropy and activation volume.

\section{Free energy sampling}

We demonstrate the free energy landscape and the long timescale collective atomic diffusion mechanism triggered by $\mathrm{Cu}$ atoms in a prototypical $\mathrm{Cu}_{50} \mathrm{Zr}_{50}$ model glass which is described by a Finnis-Sinclair type empirical interatomic potential [54]. The 3D CuZr glass model contains 4000 atoms which is prepared by a standard heating-quenching technique from liquid state to glass with a cooling rate of $10^{10} \mathrm{~K} / \mathrm{s}$. An additional sub- $T_{\mathrm{g}}$ annealing at $700 \mathrm{~K}$ is performed for $500 \mathrm{~ns}$ to thoroughly relax the structure [55]. Periodic boundary conditions are applied in each direction during both normal MD and metadynamics samplings. The MD timestep $\Delta t=2 \mathrm{fs}$.

The hopping distance of a single "tracer" $\mathrm{Cu}$ atom with respect to its initial position is adopted as the collective variable (CV) of metadynamics [56]. At each target temperature, the configuration is adopted from the cooling process with memory of velocity information. The finite temperature models are further being equilibrated for 200 ps within an isothermal-isobaric ensemble before metadynamics samplings [57]. Well-tempered metadynamics samplings [56,58] are then performed within the canonical ensemble with constant number of atoms, temperature and volume. A history-dependent bias potential is constructed on-the-fly according to the fluctuation of the hopping distance (or CV). The Gaussians are deposited every 1000 steps (each timestep $2 \mathrm{fs}$ ), with a starting height of $0.02 \mathrm{eV}$. The width of Gaussian is $0.35 \AA$ with a $0.01 \AA$ resolution. Note that there may exist an obvious length and energy scale at which the transitions take place. In a glass, these quantities are not at all clear and perhaps there might not even be such length-scales. However, the length scale is at least larger than the nearest neighbor distance of usually the order of $\sim 2 \AA(2.7 \AA$ for the present $\mathrm{Cu}_{50} \mathrm{Zr}_{50}$ glass) [59], and activation energy of the order of $\sim \mathrm{eV}$ [60]. The present choices of the shape of bias potential in terms of the Gaussian width and height are much smaller than the characteristic length and energy scale of a collective diffusion event, which guarantees reasonable quantitative estimation of the explored free energy landscape. The bias potential adding rate is gradually decreased according to a fictive temperature $T+\Delta T=3000 \mathrm{~K}$ of CV [56]. By adding bias potential, the configuration is forced to escape from the original free energy basins. Finally, the FES is reconstructed by recalling the history of the added bias potential as a function of the chosen CV. All the physical quantities are ensemble average of 20 randomly selected atoms (corresponding to 20 independent events) to include the statistical feature of the well-known inhomogeneous structure and dynamic heterogeneity in MGs. A convergence test has been done which shows that 20 samplings are good enough to derive the standard error of the average free energy barrier. While we focus on collective atomic diffusion which is triggered by $\mathrm{Cu}$ atoms, the results of collective atomic diffusion triggered by $\mathrm{Zr}$ atom are also provided in Fig. S1 of Supplemental Information (SI) for comparison, which actually leads to the same conclusion as $\mathrm{Cu}$.

Fig. 1 summarizes a typical well-tempered metadynamics samplings of diffusion triggered by $\mathrm{Cu}$ atom hopping in $\mathrm{Cu}_{50} \mathrm{Zr}_{50}$, which is sampled for $20 \mathrm{~ns}$ normal MD time $\left(2 \times 10^{7}\right.$ time steps $)$ at $300 \mathrm{~K}$. Note that the metadynamics sampling time denotes $2 \times$ $10^{7} \times \Delta t$, while the physical time should be much longer $(\sim 100 \mathrm{~s}$ at $300 \mathrm{~K}$ ) for a typical diffusion event, and it should be recalculated recalling the bias potential added to the free energy surface during sampling. Fig. 1 (a) shows the evolutions of CV. The hoppings in CV except the thermal fluctuation indicate several diffusion events. In this work, we focus on the first event (first hopping) to exclude the mutual interactions. The collective event consists of 1 ) a single $\mathrm{Cu}$ atom hopping corresponding to escaping from one energy basin with overcoming the free energy barrier and 2) many atom hoppings induced by the single $\mathrm{Cu}$ atom hopping happening because of mutual influence. The inset in Fig. 1(a) shows the trajectories of a metadynamics boosted atom (tracer) in comparison with that of a normal MD atom for the same time steps, which demonstrates the advantage of sampling capacity of metadynamics over the conventional atomistic simulations. The added bias potential is plotted in Fig. 1(b) as a function of CV. An analysis on the deposited Gaussians reproduces the original FES, as shown in Fig. 1(c). Note that the free energy landscape shown in Fig. 1(c) is roughly the inverse plot of the outer envelope of the added bias potential during well-tempered metadynamics samplings, as shown in Fig. 1(b). The free energy landscape is actually the cumulative work done by the bias potential as it moves with variation of the CV. Histograms of the single atom hopping distance $a$ and free energy 

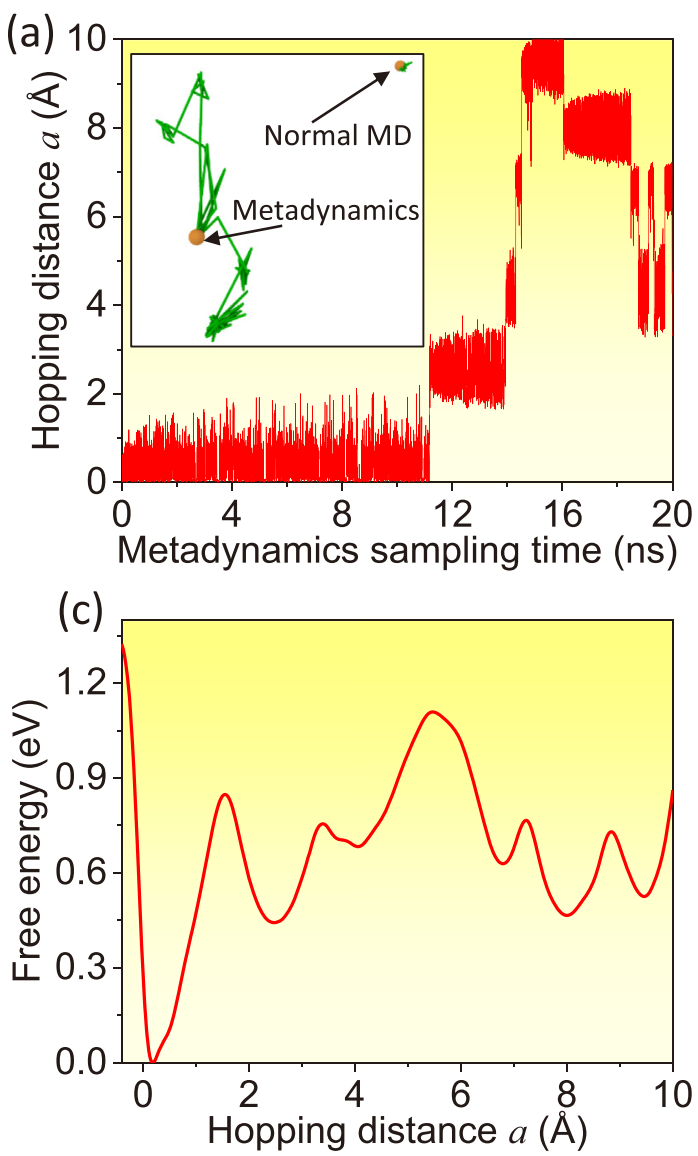
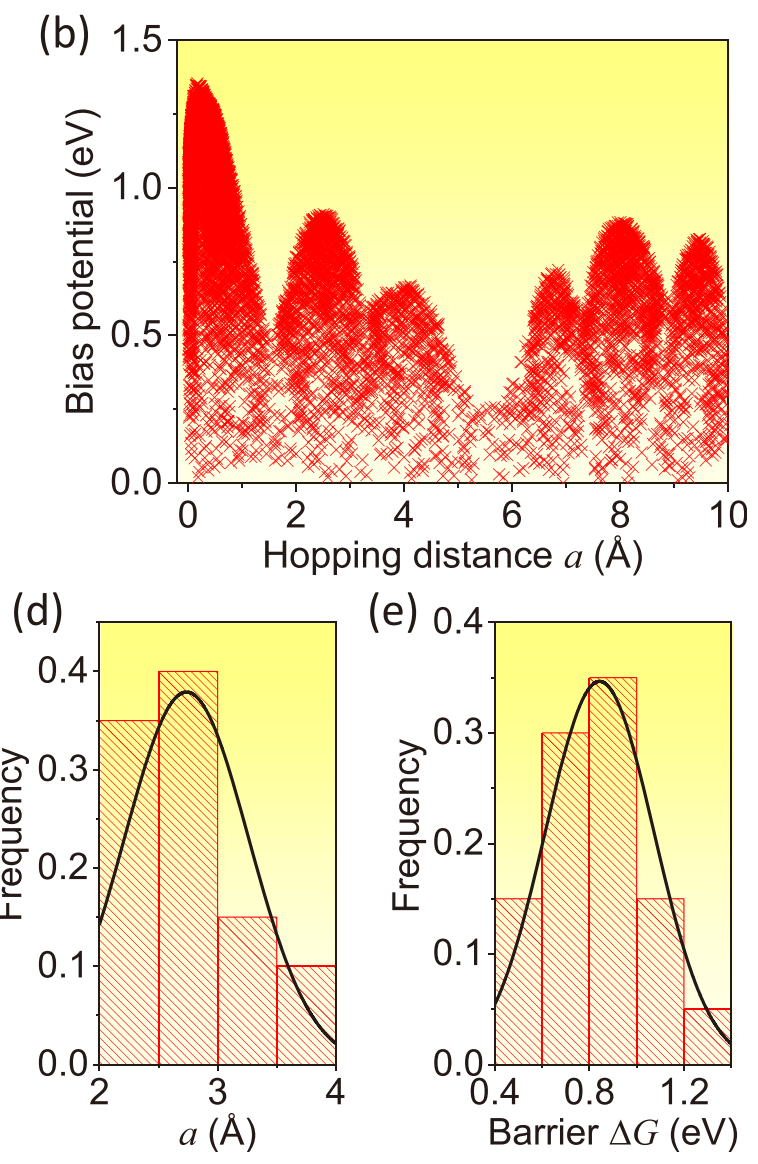

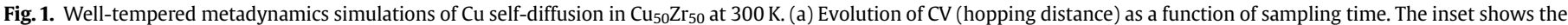

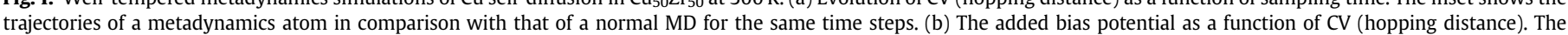

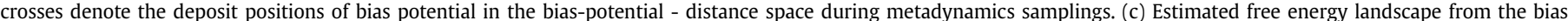
potential shown in (b). (d) Histogram of the hopping distance $a$, and (e) free energy barrier $\Delta G$ of 20 randomly selected boosted atoms.

barrier $\Delta G(300 \mathrm{~K})$ of 20 independent samplings are shown in Fig. 1 (d) and (e). At $300 \mathrm{~K}$, the hopping distance is $a=2.7 \pm 0.5 \AA$, which is of the order of the nearest neighbor distance in the present glass. This magnitude is comparable either to the experimental observations [1] or previous atomistic simulations [18,61]. The free energy barrier $\Delta G(300 \mathrm{~K})$ of diffusion is estimated between $0.53 \mathrm{eV}$ and $1.33 \mathrm{eV}$ at $300 \mathrm{~K}$, as searched within 20 independent samplings. According to the transition state theory (TST) [62], the frequency of atom hopping is predicted as $\nu=\nu_{0} \exp \left[-\frac{\Delta G(T)}{k_{\mathrm{B}} T}\right]$, where $k_{\mathrm{B}}$ is the Boltzmann constant, and $\nu_{0} \sim 3 \times 10^{12} \mathrm{~s}^{-1}$ is the attempt frequency of hopping that determined from the initial energy curvature along the FES. Therefore, the atom hopping time can be derived at each temperature with the knowledge of temperature-dependent free energy barriers.

After sampling the free energy landscape of all temperatures, the hopping time of diffusion events, i.e. $\tau=\nu^{-1}=\nu_{0}^{-1} \exp \left[\frac{\Delta G(T)}{k_{\mathrm{B}} T}\right]$, as a function of temperature are further provided in Fig. 2. The meshed area defines a possible regime of hopping time in the present laboratory timescale samplings. The spanned range of hopping time is calculated with the largest and smallest activation free energies as searched, which determine the longest waiting time $\tau_{\text {upper }}$ and shortest waiting time $\tau_{\text {lower, }}$ respectively. Note that the dynamic heterogeneity due to inhomogeneous structure is an intrinsic feature of metallic glasses, there should be diffusion events of very wide range of timescales, especially at low temperature.

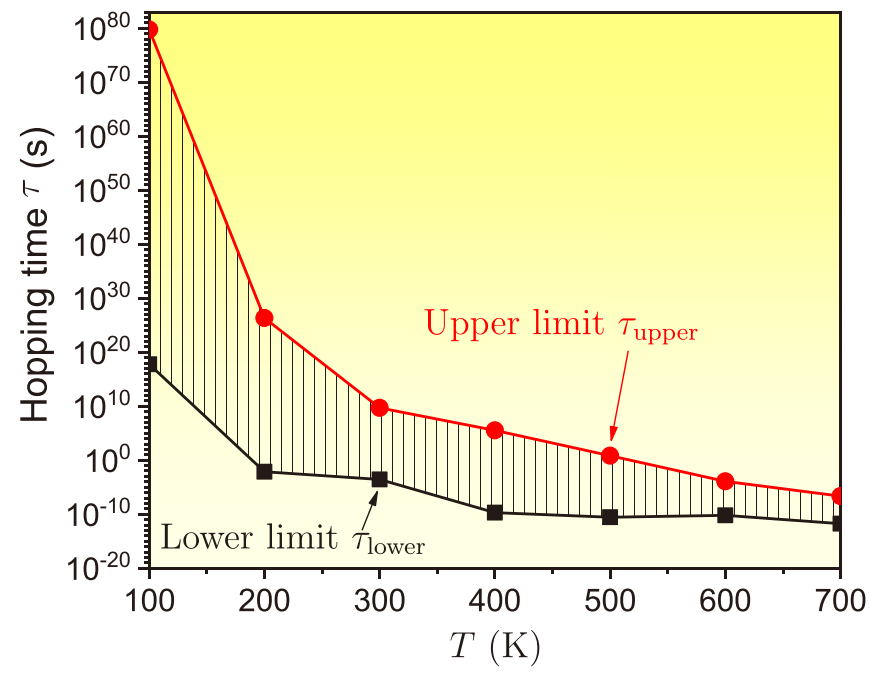

Fig. 2. The hopping incubation time of the triggered atom as a function of temperature. The shaped area is drawn by the longest and shortest physical hopping time of 20 independent metadynamics samplings at each temperature. The wide range of hopping time indicates dynamic heterogeneity of metallic glass, in particular at low temperature.

Therefore more quantitative estimation of the apparent hopping time requires more samplings because we need to figure out the 
spatial distribution of atomic hopping frequency with fine resolution, while here we demonstrate a possible regime of hopping time within the finite 20 independent samplings for simplicity. Since the samplings are still computationally expensive with about $10^{7}$ timesteps to observe diffusion events, the present strategy is accepted to capture the qualitative picture of the dynamic heterogeneity of glass. Fig. 2 shows that the hopping timescale of atoms depends strongly on temperature and spans many orders of magnitudes from $100 \mathrm{~K}$ to $700 \mathrm{~K}$. At ambient temperature, the hopping timescale ( $\sim$ second) is significantly beyond the reach of normal MD. At extremely low temperature, e.g. $200 \mathrm{~K}$, the estimated timescales of some events are already beyond the experimental observation time limit. Finally, the narrowed spanning range of hopping time at high temperature is an indication of reduced dynamic heterogeneity, which is in agreement with experimental phenomena [11]. Besides the atomic diffusion triggered by $\mathrm{Cu}$ atom, we also performed extra samplings of the diffusion events triggered by $\mathrm{Zr}$ atom (refer details to Fig. S1), which is shown to have relatively larger free energy barrier (since $\mathrm{Zr}$ atom is heavier than $\mathrm{Cu}$ atom) but comparable single atom hopping distance, which is also of the order of nearest neighbor distance. In detail, the hopping distance of the triggered $\mathrm{Zr}$ atoms is $2.6 \pm 0.9 \AA$ after 20 independent samplings on randomly selected atom at $300 \mathrm{~K}$, which is almost identical to the value of $\mathrm{Cu}$ atom, i.e., $2.7 \pm 0.5 \AA$ at the same temperature. However, its free energy barrier is estimated as $1.38 \pm 0.76 \mathrm{eV}$ at $300 \mathrm{~K}$, which is obviously larger than that of $\mathrm{Cu}(0.84 \pm 0.25 \mathrm{eV})$. It indicates that the collective diffusion events triggered by $\mathrm{Zr}$ atoms take longer time to occur since $\mathrm{Zr}$ atom is heavier than $\mathrm{Cu}$ atom. But the collective atomic diffusion mechanism remains unchanged no matter if the diffusion is triggered either by $\mathrm{Cu}$ atom or $\mathrm{Zr}$ atom.

\section{Results and discussion}

\subsection{Quantify the mechanism of collective atomic diffusion}

Fig. 3(a) demonstrates the diffusion patterns from extremely low temperature $1 \mathrm{~K}$ to high temperature $700 \mathrm{~K}$, which has already touched the thermal glass transition temperature $\left(T_{\mathrm{g}}=690 \mathrm{~K}\right.$ estimated from the present interatomic potential). Only the hopped atoms more than $0.5 \AA$ hopping triggered by the single atom hopping have been shown by comparing the inherent structures before and after hopping, respectively. The inherent structure is a structure in local energy minimum of potential energy surface [63]. The arrows represent the displacement vectors of the hopped atoms. Note that the configurations in Fig. 3(a) are one typical example of the 20 independent samplings at each target temperature. The atomic diffusion events consist of multiple atomic hopping. They are shown to be localized string-like cooperative motion in the glassy state, agreeing with previously findings explored by normal MD [12,32-35]. The diffusion string becomes longer and longer, and finally presents 3D characteristics. Since the inherent structures changes very little with variation in temperature (shown in Fig. S2), one may attribute the transition of diffusion mechanisms to the critical role of temperature in modulating the diffusion free energy landscape. Although the displacement field of the collective event is noisy at high temperatures, the minimum energy reactive pathway is of a normal single-peak profile, as shown in Fig. S3 of SI. It indicates that the collective event is a real hopping between neighboring basins in the energy landscape.

There still leaves an open question that why only one atom excitation can be a trigger of such string-like local structural excitation (LSE) consisting of many atom hoppings, and whether such string-like LSE can happen anywhere. By further examination of the searched minimum energy reactive pathways using nudged elastic band method (NEB) (as shown in Fig. S3 of SI), we observe an interesting mechanism of downhill dynamics corresponding to the string-like collective atomic motion, and this can happen anywhere. Most fraction of the event is after the saddle point, while the uphill activation involves only few atoms motion including the triggered atom. As a result, the whole event can be induced by only encouraging hopping of the triggered atom in the well-tempered metadynamics samplings.

In Fig. 3(b), the mean distance of hopping of the triggered atom becomes slightly shorter with increasing temperature; see also the scattered plot in Fig. S4 of SI. However, free energy barriers become smaller at high temperature $[18,61]$. Instead of the hopping distance of the triggered single atom, the $l^{2}$-norm which is the Euclidean norm, can be utilized to better quantify the total diffusion distance in the $3 \mathrm{~N}$-dimensional configuration space, which is a kind of "size" of the collective atomic diffusion event triggered by a single atom hopping. The $l^{2}$-norm of a collective atomic diffusion event is defined as (a)

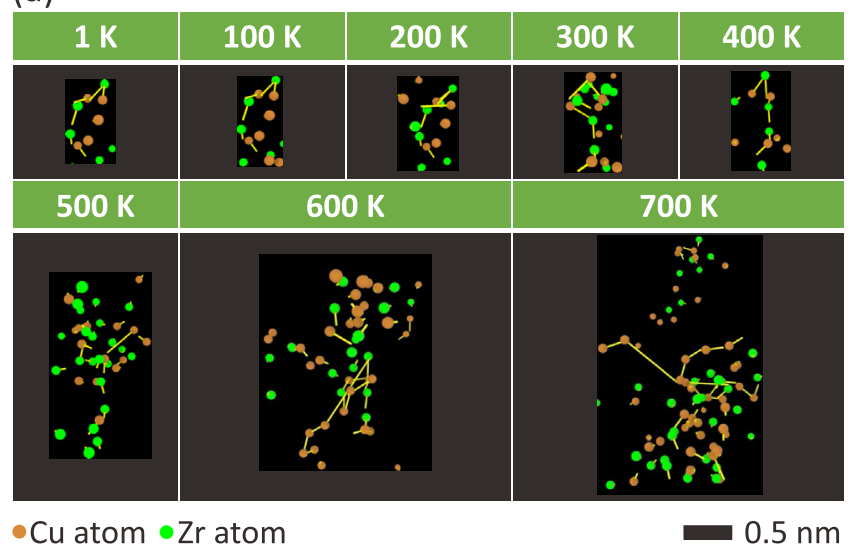

(b)

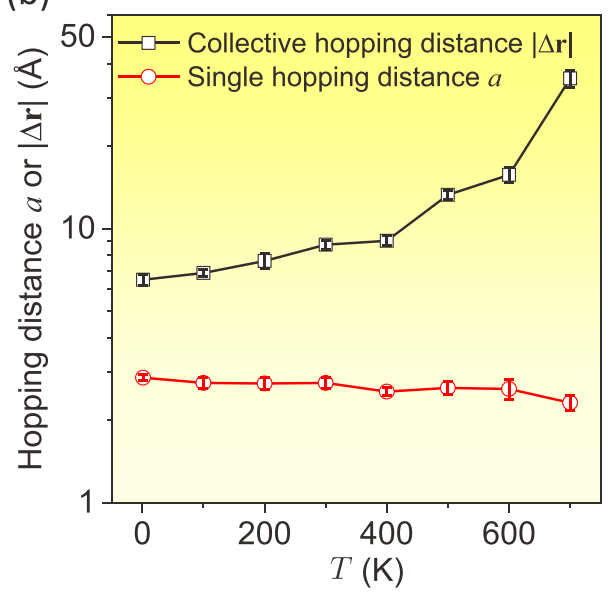

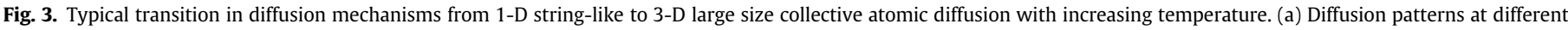

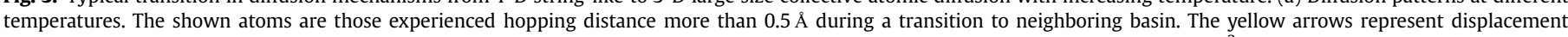

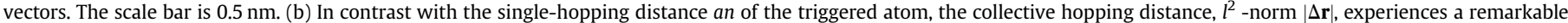

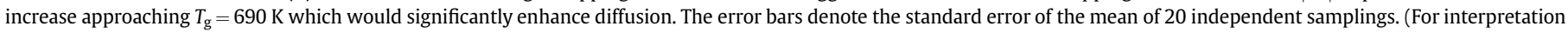
of the references to colour in this figure legend, the reader is referred to the Web version of this article.) 
$|\Delta \mathbf{r}|=\sqrt{\sum_{i=1}^{N}\left(\Delta \mathbf{r}_{i}^{2}\right)}=\sqrt{N \cdot \mathrm{MSD}}$

where $i$ is the ID of atoms, $\Delta \mathbf{r}_{i}$ the displacement vector of atom $i, N$ the total number of atoms, and MSD short for the usual concept of mean squared displacement of all atoms, i.e., $\operatorname{MSD}=\sum_{i=1}^{N}\left(\Delta \mathbf{r}_{i}^{2}\right) / N$. $|\Delta \mathbf{r}|$ is obtained by comparing the inherent structures of the initial free energy minimum and that of its neighboring basin, respectively. Note that $|\Delta \mathbf{r}|$ is mathematically similar to the root mean squared displacement (RMSD). However, it compares two inherent structures (before and after hopping) that already excludes the thermal fluctuation, which is quantitatively meaningful to characterize the size of a bare collective atomic diffusion event.

In Fig. 3(b) we plot the single atom hopping distance of the boosted atom $a$ and $l^{2}$-norm of the whole collective atomic diffusion event as a function of temperature. Instead of the very slight decrease in single atom hopping distance, $|\Delta \mathbf{r}|$ increases moderately with increasing temperature up to $400 \mathrm{~K}$. This is to say that the size of the 1-D string-like collective atomic diffusion does not change substantially. It is in agreement with the consensus of mode-coupling theory that MSD experiences a plateau below the MCT critical temperature $T_{\mathrm{c}}$ due to the well-known cage effect of local rattling $[3,31,64]$. The MSD then increases as the relaxational motions begin to set in, as evidenced from the neutron scattering experiments [64]. In our case, $|\Delta \mathbf{r}|$ increases very rapidly after a temperature between $400 \mathrm{~K}$ and $500 \mathrm{~K}$. This is maybe corresponding to the transition from 1-D string to 3-D large diffusion as shown in Fig. 3(a). The diffusion event becomes larger at higher temperature with participation of more atoms that contributes significantly to MSD (as well as diffusivity). It seems that the collective atomic diffusion nature in glass transforms from 1-D (local) to 3-D (unlocal) upon approaching the thermal glass transition.

\subsection{Estimation of collective diffusivity}

Under the assumption that diffusion is a thermally activated process below the MCT critical temperature [3,7,17], the diffusion kinetics can be understood with the knowledge of the activation Gibbs free energy,

$\Delta G(T, P)=\Delta Q+P V-T \Delta S$,

where $P$ is hydrostatic pressure, $V$ the activation volume, and $\Delta Q$ the activation energy at condition of $P=0$ and $T=0 . \Delta H=\Delta Q+P V$ is the activation enthalpy, and $\Delta S$ the activation entropy. TST consequently predicts the temperature- and pressure-dependent diffusivity as [62] [3],

$D(T, P)=g f a_{\mathrm{eff}}^{2} \nu_{0} \exp \left[-\frac{\Delta G(T, P)}{k_{\mathrm{B}} T}\right]$,

which significantly depends on the effective hopping distance of the collective atomic motion $a_{\mathrm{eff}}=\sqrt{\sum_{i=1}^{n}\left(\Delta \mathbf{r}_{i}^{2}\right)} \approx|\Delta \mathbf{r}|$ besides the energy barrier, where $n$ is the number of the travelled atoms except the thermal fluctuation, as those shown in Fig. 3(a). Note that here we have assumed the diffusivity is mainly contributed by the travelled atoms which have experienced real hopping from one energy basin to another neighboring basin. Thermal fluctuation has been excluded in determination of $|\Delta \mathbf{r}| . g=1 / 6$ is a geometrical factor for the 3 -D diffusion [2,3]. $0<f \leq 1$ is a correlation factor of the order of unity for diffusion in metallic glasses [1,65]. The sudden increase of $|\Delta \mathbf{r}|$ and the number of diffusing atoms approaching $T_{\mathrm{g}}$ probably rationalizes the frequently experimental observation of non-Arrhenius kink around $T_{\mathrm{g}}$ [1], while the hopping frequency of single atom remains Arrhenius-like which is thoroughly governed by the free energy barrier, i.e., $\nu \propto \exp \left[\Delta G(T, P) / k_{\mathrm{B}} T\right]$. It is the abrupt increase in size of the diffusion event that plays a dominating role in the enhanced diffusivity, as we confirmed here in Fig. 3. The results are consistent with a recent demonstration of the transition from localized process in the slowly quenched glass to cascade deformation in the fast quenched metallic glass sample [18]. While the cascade deformation involves more atoms since a higher density of local potential energy basins appear in the less stable glassy structure, as explained by Fan et al. in Refs. [61] and [18], in analogy to the high temperature cases of our metadynamics samplings.

After metadynamics samplings, all the parameters used to calculate the diffusivity are listed in Table 1, shown in the case of $300 \mathrm{~K}$ as an example. The average hopping distance of single atom is $a=2.7 \pm 0.5 \AA$ as explained in Fig. 3, which is of the order of the nearest neighbor distance. If the diffusion mechanism involves only one atom, then the hopping distance should be similar to such a nearest-neighbor distance. However, the diffusion in metallic glass is collective in nature. As a result, the effective hopping distance of a collective diffusion is $a_{\mathrm{eff}}=|\Delta \mathbf{r}|=8.7 \AA$, which is much larger than the single atom hopping distance. In the $3 \mathrm{~N}$-dimensional configurational space, the squared hopping distance is $a_{\mathrm{eff}}^{2}$, which is one order of magnitude larger than $a^{2}$ at $300 \mathrm{~K}$. In particular at high temperature (e.g. $700 \mathrm{~K}$ ), one may anticipate a huge difference in diffusion size between collective diffusion $\left(a_{\text {eff }}^{2} \sim 1600 \AA^{2}\right)$ and single atom diffusion $\left(a^{2} \sim 7 \AA^{2}\right)$. Consequently, there should be a transition in diffusivity due to the abrupt increase in $a_{\text {eff }}^{2}$ which is beyond the conventional Arrhenius law as presented in crystal diffusion. This is likely to the atomic-scale mechanism underlying the non-Arrhenius diffusivity upon glass transition. In summary, an increasing diffusivity with temperature can be the result of (i) atoms hopping further, (ii) atoms hopping at a higher rate, (iii) more atoms hopping; or any combination of these three. Obviously (i) is not true as shown in Fig. 3(b), (ii) is trivial and therefore true, so what we see is the presence of effect (iii): more atoms hopping. This last effect can be easily explained by the glassy structure. In a glass there is a whole spectrum of activation energies available for atomic hoppings (also if executed by groups of atoms rather than by single atoms) [18,61]; as temperature increases, more and more of the higher-energy barriers become crossable, and hence more and more atoms take part in the diffusion.

At $300 \mathrm{~K}$, the sampled free energy barriers of 20 events are calculated as between $0.53-1.33 \mathrm{eV}$. Consequently, we estimate the lower limit and upper limit of diffusivity as $D(300 \mathrm{~K})=2.5 \times 10^{-29}$ $\mathrm{m}^{2} / \mathrm{s}$, and $4.8 \times 10^{-16} \mathrm{~m}^{2} / \mathrm{s}$, respectively. The upper limit implies an existence of a weakest link of diffusion pathway, such as a link of low energy saddle points of hopping in FES, in the inhomogeneous structure and dynamic heterogeneity of MGs, while lower limit

Table 1

Parameters for estimation of diffusivity predicted by well-tempered metadynamics at $300 \mathrm{~K}$.

\begin{tabular}{lll}
\hline Physical quantity & Symbol & Value \\
\hline Geometric factor & $g$ & $1 / 6[3]$ \\
Correlation factor & $f$ & $\sim 1[65]$ \\
Effective hopping distance & $a_{\mathrm{eff}}$ & $8.7 \AA$ \\
Attempt frequency & $\nu_{0}$ & $3.0 \times 10^{12} \mathrm{~s}^{-1}$ \\
Free energy barrier & $\Delta G$ & $0.53-1.33 \mathrm{eV}$ \\
Activation entropy & $\Delta S$ & $(19.6 \pm 2.5) k_{\mathrm{B}}$ \\
Pre-exponential factor & $D_{0}$ & $1.2 \times 10^{2} \mathrm{~m}^{2} / \mathrm{s}$ \\
Diffusion coefficient & $D$ & $2.5 \times 10^{-29}-4.8 \times 10^{-16} \mathrm{~m}^{2} / \mathrm{s}$ \\
\hline
\end{tabular}


implies the weakest link is interrupted and subdivided by high energy saddle points. The scope of diffusivity is of about 10 orders of magnitude slower than the simulating capacity of the normal MD. The metadynamics predicted diffusivity is comparable to usual experimental data [3]. The diffusivity prefactor $D_{0}=g f a_{\text {eff }}^{2} \nu_{0} \exp \left(\Delta S / k_{\mathrm{B}}\right)=1.2 \times 10^{2} \mathrm{~m}^{2} / \mathrm{s}$ is also fairly within the range of experimental data range $\left(10^{-15}-10^{15} \mathrm{~m}^{2} / \mathrm{s}\right)$, considering the large scattered values of the activation enthalpy for diffusion of glasses, as summarized in Ref. [3]. Large prefactor of diffusion coefficient indicates strong entropic effect on collective atomic diffusion. The critical role of activation entropy played in collective atomic diffusion will be explained later when the temperature dependence of FES is discussed.

Fig. 4 shows the Arrhenius plot of diffusivity at wide temperature range $(100-700 \mathrm{~K})$, including the regime of metadynamics prediction, normal MD data at high temperature, as well as experimental data for several metallic glasses. For reference, $T_{\mathrm{g}}=690 \mathrm{~K}$ for the present chemical composition of the model glass. In the general disorder materials, there should display a broad distribution of energy barriers [18,61,66-68]. Given such a wide spectrum, i.e. assuming the probability distribution function of free energy barriers is $P(\Delta G)$, where $\int_{0}^{\infty} P(\Delta G) \mathrm{d} \Delta G=1$, one can define an effective free energy barrier $\overline{\Delta G}$ by assuming that the apparent event frequency is the summation of individual event frequencies, i.e. $\exp \left(-\frac{\overline{\Delta G}}{k_{\mathrm{B}} T}\right)=\int_{0}^{\infty} P(\Delta G) \exp \left(-\frac{\Delta G}{k_{\mathrm{B}} T}\right) \mathrm{d} \Delta G$. This assumes a parallel diffusion, $\overline{\Delta G}_{\text {eff }}^{\text {Para }}=-k_{\mathrm{B}} T \ln \left[\int_{0}^{\infty} P(\Delta G) \exp \left(-\frac{\Delta G}{k_{\mathrm{B}} T}\right) \mathrm{d} \Delta G\right]$ [68].

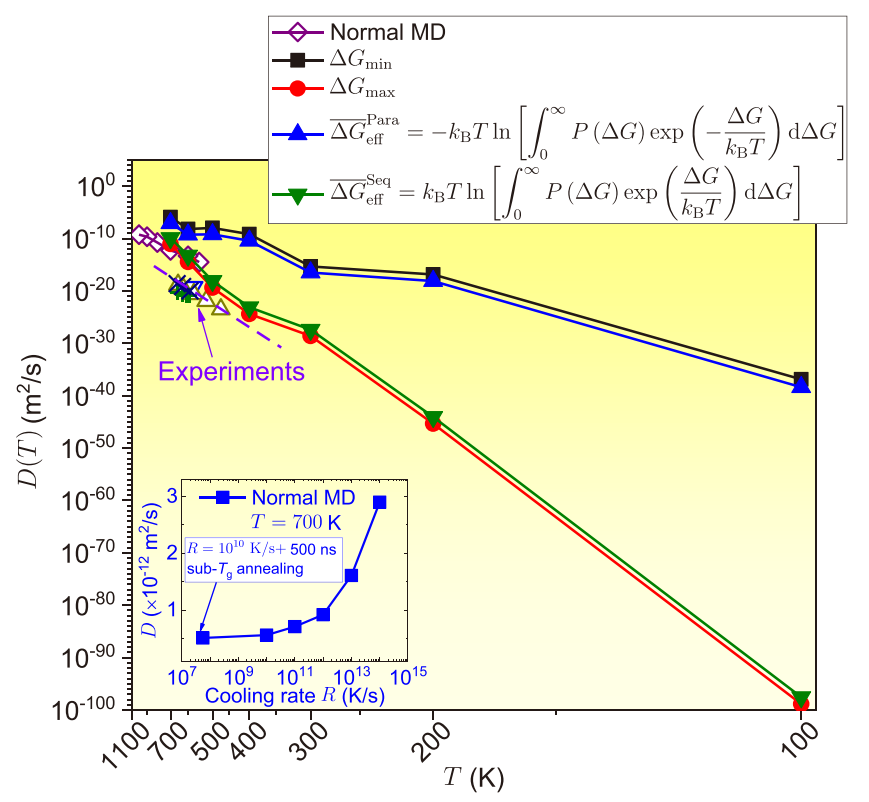

Fig. 4. Arrhenius plot of diffusivity accommodated by collective atomic motion in $\mathrm{Cu}_{50} \mathrm{Zr}_{50}$. The four lines with solid symbols define a possible regime of diffusivity predicted by the well-tempered metadynamics samplings, based on different definitions of effective activation free energies, i.e. the minimum barrier $\Delta G_{\min }$, the maximum barrier $\Delta G_{\max }$, the effective barrier with the assumption of parallel diffusion $\overline{\Delta G}_{\text {eff }}^{\text {Para }}$, and the effective barrier with the assumption of sequential diffusion $\overline{\Delta G}_{\text {eff }}^{\text {Seq }}$. The open rhombi represent normal MD estimation, which benchmarks the metadynamics calculations at high temperature. Experimental diffusivity of different glasses are shown by open symbols. The dashed line denotes an Arrhenius plot with $\Delta Q_{\exp }=1.51$ $\mathrm{eV}$ for experimental diffusivity. Metadynamics overestimates the experimental diffusivity since the shallow basins in free energy landscape of MD sample (highly rejuvenated state). The inset indicates the slowed down diffusivity with decreasing cooling rate in glassy structure preparation.
Thus, even some events are almost killed by very high free energy barriers, the other events having low free energy barriers can still be activated and almost maintain the apparent frequency. In this assumption, the diffusion event with having the lowest energy barrier dominates the apparent diffusivity. One the other hand, we can also define a sequential diffusion by assuming that the apparent diffusion event incubation time is the summation of individual events incubation time. In this case, there is no bypass route when the event encounters very high free energy barrier. The effective barrier can be written as $\overline{\Delta G}_{\text {eff }}^{\text {Seq }}=k_{\mathrm{B}} T \ln \left[\int_{0}^{\infty} P(\Delta G) \exp \left(\frac{\Delta G}{k_{\mathrm{B}} T}\right) \mathrm{d} \Delta G\right]$. In this assumption, since the high free energy barrier should be overcome for successive diffusion, the diffusion event having the highest energy barrier dominates the apparent diffusivity. There are four solid lines in Fig. 4 which denote diffusivities predicted by different definitions of effective barriers of the samplings. The minimum barrier $\Delta G_{\text {min }}$ of samplings leads to the upper limit of diffusivity, while the maximum barrier $\Delta G_{\max }$ yields the lower limit of diffusivity. The diffusivities based on the effective barriers of parallel and sequential diffusion nature are always mathematically within the regime defined by the upper and lower limit of diffusivity.

To benchmark the metadynamics calculations, we also plot the diffusivity calculated by normal $\mathrm{MD}$ at high temperature (550-900 K). The normal MD data are calculated according to the Einstein equation that the diffusivity is measured as the slope of MSD as a function of time. The temperature range here is likely below the MCT critical temperature $T_{\mathrm{c}}$ (well above $T_{\mathrm{g}}$ ), where Arrhenius relationship is assumed to be applicable according to the MCT [3,7,17]. The metadynamics diffusivity based on $\overline{\Delta G}_{\text {eff }}^{\text {Seq }}$ is in agreement with the normal MD data. It indicates the sequential scenario is a reasonable assumption of diffusion in metallic glass, at least at high temperature where conventional MD is statistically reliable. We believe that the sequential scenario is true even at lower temperature, while further study for dynamic heterogeneity, such as the details of spatial distribution of atomic hopping frequency, is necessary to confirm it. To partially support this scenario, we further provide the spatial distribution of free energy barriers of a deep glassy structure at $0 \mathrm{~K}$ in Fig. S5 of SI. One can notice that the low energy barrier regions (preferred diffusion locations) are frequently surrounded by high barrier regions, which means easy diffusion pathways are intermediately interrupted and consequently the effective diffusivity should take the high energy barriers into consideration. Therefore, the assumption of parallel diffusion may not be applicable to metallic glasses.

An Arrhenius fit of the metadynamics data yields the apparent activation energies of $\Delta Q_{\text {lower }}=0.70 \mathrm{eV}$, and $\Delta Q_{\text {upper }}=1.99 \mathrm{eV}$, respectively, for the upper and lower limit of diffusivity. For a qualitative comparison, the calculated activation energy is in line with the available experimental data range, e.g., $1.33 \mathrm{eV}$, and $1.57 \mathrm{eV}$ for $\mathrm{Cu}$ diffusion in $\mathrm{Zr}_{61} \mathrm{Ni}_{39}$ [69], and $\mathrm{Zr}_{50} \mathrm{Ni}_{50}$ [70], respectively. In $\mathrm{Cu}_{50} \mathrm{Zr}_{50}$, the activation energy of $\mathrm{Ag}$, and Au diffusion is measured as 0.82 , and $1.55 \mathrm{eV}$, respectively [71]. The experimental diffusivity are also shown in Fig. 4. Metadynamics overestimates diffusivity for several orders of magnitudes. Besides the difference in glass composition and the tracer atoms, the discrepancy is also possibly attributed to the less stable MD glassy structure than those of experiments. The MD samples are prepared with a fast cooling rate which leads to an unstable energetic state with a relatively low relaxed shear modulus [60] and a shallow mega-basin [18,61]. This assumption is verified by the slowed down diffusivity with decreasing cooling rate of glass preparation, as demonstrated in the inset of Fig. 4. The dashed line in Fig. 4 denotes an Arrhenius plot of diffusivity with a hypothetic apparent activation energy of 
$\Delta Q_{\text {exp }} \approx 26 k_{\mathrm{B}} T_{\mathrm{g}}=1.51 \mathrm{eV}$ [10], which matches the experimental diffusivity as anticipated. Therefore, it is concluded that the overestimation of diffusivity is due to the hollow basin of unstable computer glassy structure.

\subsection{Temperature and pressure dependence}

The temperature and pressure effect on free energy landscape are summarized in Fig. 5. In Fig. 5(a) we show three representative FES at 1,300 , and $700 \mathrm{~K}$, respectively. The free energy barrier is effectively reduced by increasing temperature. The CV is also affected by temperature. At high temperature, more local basin is presented with smaller barriers; see a log-log scatter plot of barriers versus hopping distance in Fig. S4 of SI. Fig. 5(b) plots $\overline{\Delta G}_{\text {eff }}^{\text {Seq }}$ of 20 samplings versus $T$. Here we choose the sequential effective barrier $\overline{\Delta G}_{\text {eff }}^{\text {Seq }}$ at each temperature, since it yields the effective diffusivity which is comparable to the normal MD estimation of diffusivity at high temperature, as shown in Fig. 4. The free energy barrier decreases almost linearly with increasing temperature. The slope leads to a temperature insensitive activation entropy of the order of

$\Delta S \equiv-\frac{\partial \Delta G}{\partial T}=(19.6 \pm 2.5) k_{\mathrm{B}}$

according to Eq. (2). The derived activation entropy agrees with the experimental data $[3,8]$. For activation enthalpy ranging $1-3 \mathrm{eV}$, the activation entropy could be $19 k_{\mathrm{B}}$ to $56 k_{\mathrm{B}}$ [3]. If diffusivity is expressed as, $D=D_{0} \exp \left(-\frac{\Delta H}{k_{\mathrm{B}} T}\right)$, then the pre-factor is $D_{0}=g f a_{\text {eff }}^{2} \nu_{0} \exp \left(\frac{\Delta S}{k_{\mathrm{B}}}\right)$ [3]. Considering the pronounced entropic effect, the pre-factor of diffusion coefficient is estimated to be $\sim 1.2 \times 10^{2} \mathrm{~m}^{2} / \mathrm{s}$, as shown in Table 1 . Since $\Delta S$ is not significantly temperature dependent, the experimentally found kink in Arrhenius plot around $T_{\mathrm{g}}$ should be attributed to the strong temperature dependence of the effective hopping distance $a_{\text {eff }}=|\Delta \mathbf{r}|$ as appeared in Eq. (3). The hypothesis is confirmed by the temperature induced transition of diffusion mechanisms as the patterns
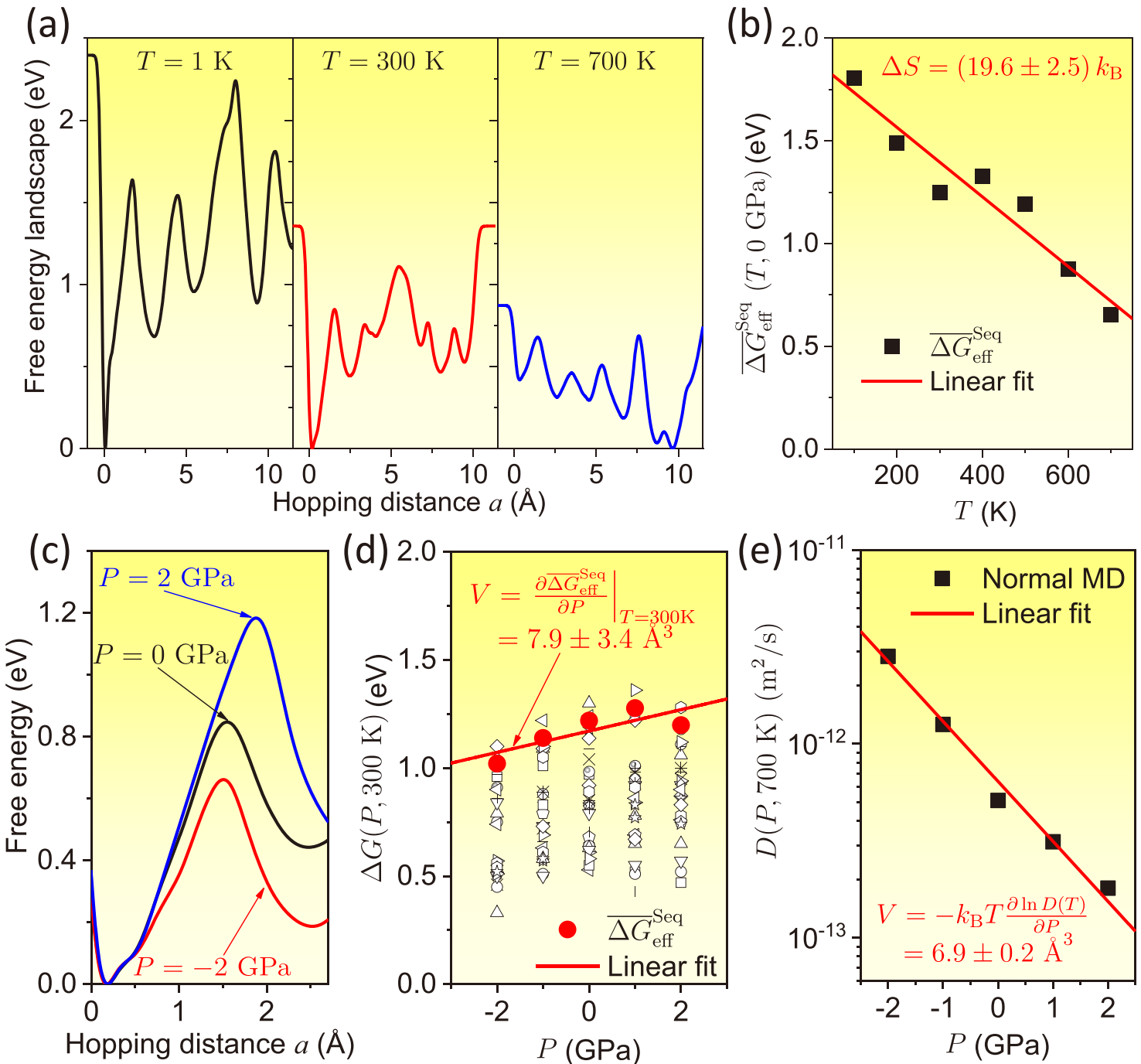

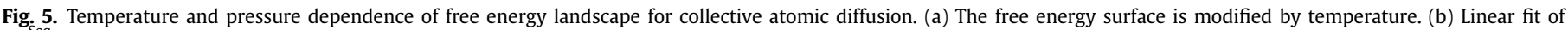

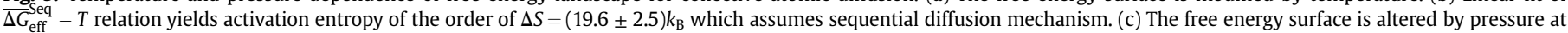

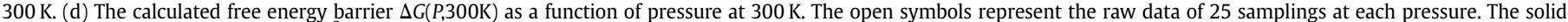

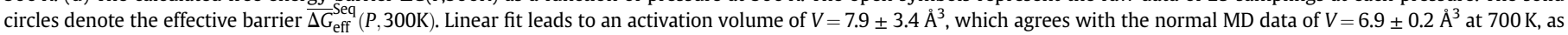
shown in (e) 
shown in Fig. 3. Note that $P(\Delta G)$ is not fixed at different temperatures due to maybe the vibrational entropic effect, which is confirmed by the distribution of free energy barriers shown in Fig. S6 at different temperatures.

Fig. 5(c-e) demonstrates the pressure effect on FES of collective atomic diffusion. Fig. 5(c) shows that pressure increases the free energy barrier. It means that one may manipulate pressure to control the dynamics of glasses. Fig. 5(d) shows the effective barrier $\overline{\Delta G}_{\text {eff }}^{\text {Seq }}$ of five different atoms triggered diffusion events considering the spatially heterogeneous structure. Each event also includes five independent samplings at the same pressure. The applied hydrostatic pressure is between -2 and $2 \mathrm{GPa}$. It seems reasonable to assume a linear relationship between $\overline{\Delta G}_{\text {eff }}^{\text {Seq }}$ and $P$, which yields an activation volume of

$V \equiv \frac{\partial \Delta G}{\partial P}=(7.9 \pm 3.4) \AA^{3}$,

according to Eq. (2). This value is $V=(0.46 \pm 0.20) \Omega_{\text {atom, with }}$ $\Omega_{\text {atom }}=17.3 \AA^{3}$ the average atomic volume in the present CuZr glass. For comparison, Fig. 5(e) shows the results at $700 \mathrm{~K}$, which is available within the direct normal MD timescale. It predicts the value of activation volume as [3] [2],

$V=-k_{\mathrm{B}} T \frac{\partial \ln D(T, P)}{\partial P}=(6.9 \pm 0.2) \AA^{3}$,

according to Eq. (2) and Eq. (3). The magnitude is about $0.5 \Omega_{\mathrm{atom}}$ which is much smaller than the activation volume of shear transformations [72]. Our simulations are in agreement with previous MD calculations showing $V=0.43 \Omega_{\text {atom }}$ for $\mathrm{Ni}$ diffusion in $\mathrm{Ni}_{50} \mathrm{Zr}_{50}$ [33]. The activation volumes from both metadynamics and normal MD are self-consistent, and they also agree quantitatively with the experimental data [3]. For example, the activation volume is experimentally determined as $V=0.5 \Omega_{\text {atom }}$ for $\mathrm{Hf}$, Au diffusion in $\mathrm{Ni}_{54} \mathrm{Zr}_{46}$ [73]; $V=(0.35-0.64) \Omega_{\text {atom }}$ for ${ }^{63} \mathrm{Ni}$ in $\mathrm{Zr}-\mathrm{Cu}-\mathrm{Ni}-\mathrm{Ti}-\mathrm{Be}$ [74]; and $(0.66 \pm 0.15) \Omega_{\text {atom }}$ for Ni diffusion in $\mathrm{Co}_{42} \mathrm{Zr}_{58}$ [75]. Finally, we note that even though large collective atomic diffusion happens, it is a result of a single triggered atom hopping process. So that we can estimate the thermodynamic values (activation entropy, volume) using the calculated $\Delta G$ (free energy barrier of a single atom hopping). The strategy is confirmed by the agreement between the calculated thermodynamic parameters and those from experiments.

\section{Conclusion}

The physical nature of collective atomic diffusion mechanism in amorphous solids are explored by the free energy samplings with atomic details in the framework of well-tempered metadynamics. Unveiled diffusive atomic motion suggests a temperaturedependent 1D string-like motion in deep glassy state, with increasing size at higher temperature, and finally transitions to 3D large size collective atomic diffusion. The finding explains the extraordinarily enhanced diffusivity once the temperature is near the glass transition. By comparing with normal MD diffusivity at high temperature, the apparent diffusivity computed from the distribution of free energy barrier of atom hopping seems to be dominated by the highest free energy barrier among them, which suggests the sequential nature of diffusion is proper assumption to the metallic glasses which have the dynamic heterogeneity. The kinetics of collective atomic diffusion are quantitatively examined by testing the temperature and pressure dependence of diffusion free energy landscape, which yields experimentally calibrated values of activation entropy and activation volume. The activation parameters benchmark the rationality of the metadynamics samplings. The present calculations provide experimentally relevant timescale observation of diffusion at atomic-scale which bring comprehensive insights into the physical mechanism of collective atomic diffusion as unique fundamental process of atom motion in amorphous solids, which is beyond the knowledge wellestablished in diffusion of crystals.

\section{Acknowledgments}

Insightful discussions with professors Jeppe C. Dyre, Peter Harrowell and Corey S. O'Hern are acknowledged. The work is financially supported by the NSFC (Nos. 11672299, 11790292), the National Key Research and Development Program of China (Nos. 2017YFB0702003, 2017YFB0701502), and the Strategic Priority Research Program of the Chinese Academy of Sciences (No. XDB22040303). S.O. acknowledges the support by JSPS KAKENHI Grant Nos. JP17H01238 and JP17K18827, and the Elements Strategy Initiative for Structural Materials (ESISM). The results described in this paper (partially) are obtained on the "Era" petascale supercomputer of Computer Network Information Center of Chinese Academy of Sciences.

\section{Appendix A. Supplementary data}

Supplementary data related to this article can be found at https://doi.org/10.1016/j.actamat.2018.07.029.

\section{References}

[1] X.P. Tang, U. Geyer, R. Busch, W.L. Johnson, Y. Wu, Diffusion mechanisms in metallic supercooled liquids and glasses, Nature 402 (1999) 160-162.

[2] H. Mehrer, Diffusion in Solids: Fundamentals, Methods, Materials, Diffusioncontrolled Processes, Springer Series in Solid-State Sciences, Springer, 2007.

[3] F. Faupel, W. Frack, M.-P. Macht, H. Mehrer, V. Naundorf, K. Rätzke, H.R. Schober, S.K. Sharma, H. Teichler, Diffusion in metallic glasses and supercooled melts, Rev. Mod. Phys. 75 (2003) 237.

[4] J. Bokeloh, S.V. Divinski, G. Reglitz, G. Wilde, Tracer measurements of atomic diffusion inside shear bands of a bulk metallic glass, Phys. Rev. Lett. 107 (2011) 235503.

[5] J. Brillo, A.I. Pommrich, A. Meyer, Relation between self-diffusion and viscosity in dense liquids: new experimental results from electrostatic levitation, Phys. Rev. Lett. 107 (2011) 165902.

[6] V. Chikkadi, D.M. Miedema, M.T. Dang, B. Nienhuis, P. Schall, Shear banding of colloidal glasses: observation of a dynamic first-order transition, Phys. Rev. Lett. 113 (2014) 208301.

[7] S.W. Basuki, A. Bartsch, F. Yang, K. Rätzke, A. Meyer, F. Faupel, Decoupling of component diffusion in a glass-forming $\mathrm{Zr}_{46.75} \mathrm{Ti}_{8.25} \mathrm{Cu}_{7.5} \mathrm{Ni}_{10} \mathrm{Be}_{27.5}$ melt far above the liquidus temperature, Phys. Rev. Lett. 113 (2014) 165901.

[8] F. Strauß, L. Dörrer, T. Geue, J. Stahn, A. Koutsioubas, S. Mattauch, H. Schmidt Self-diffusion in amorphous silicon, Phys. Rev. Lett. 116 (2016) 025901.

[9] D. Rodney, C. Schuh, Distribution of thermally activated plastic events in a flowing glass, Phys. Rev. Lett. 102 (2009) 235503.

[10] H.B. Yu, K. Samwer, Y. Wu, W.H. Wang, Correlation between $\beta$ relaxation and self-diffusion of the smallest constituting atoms in metallic glasses, Phys. Rev. Lett. 109 (2012) 095508.

[11] Z. Wang, B.A. Sun, H.Y. Bai, W.H. Wang, Evolution of hidden localized flow during glass-to-liquid transition in metallic glass, Nat. Commun. 5 (2014) 5823.

[12] H.B. Yu, R. Richert, K. Samwer, Structural rearrangements governing joharigoldstein relaxations in metallic glass, Sci. Adv 3 (2017), e1701577.

[13] S.V. Ketov, Y.H. Sun, S. Nachum, Z. Lu, A. Checchi, A.R. Beraldin, H.Y. Bai, W.H. Wang, D.V. Louzguine-Luzgin, M.A. Carpenter, A.L. Greer, Rejuvenation of metallic glasses by non-affine thermal strain, Nature 524 (2015) 200-203.

[14] N. Miyazaki, M. Wakeda Y.J. Wang S. Ogata Prediction of pressurepromoted thermal rejuvenation in metallic glasses, npj Comput. Mater. 2 (2016) 16013

[15] J. Pan, Y.X. Wang, Q. Guo, D. Zhang, A.L. Greer, Y. Li, Extreme rejuvenation and softening in a bulk metallic glass, Nat. Commun. 9 (2018) 560.

[16] J. Zemp, M. Celino, B. Schönfeld, J.F. Löffler, Crystal-like rearrangements of icosahedra in simulated copper-zirconium metallic glasses and their effect on mechanical properties, Phys. Rev. Lett. 115 (2015) 165501.

[17] V. Zöllmer, K. Rätzke, F. Faupel, A. Meyer, Diffusion in a metallic melt at the critical temperature of mode coupling theory. Phys. Rev. Lett. 90 (2003) 195502. 
[18] Y. Fan, T. Iwashita, T. Egami, Crossover from localized to cascade relaxations in metallic glasses, Phys. Rev. Lett. 115 (2015) 045501.

[19] D.Z. Chen, C.Y. Shi, Q. An, Q.S. Zeng, W.L. Mao, Fractal atomic-level percolation in metallic glasses, Science 349 (2015) 1306-1310.

[20] I.R. Peters, S. Majumdar, H.M. Jaeger, Direct observation of dynamic shear jamming in dense suspensions, Nature 532 (2016) 214-217.

[21] C. Schuh, T. Hufnagel, U. Ramamurty, Mechanical behavior of amorphous alloys, Acta Mater. 55 (2007) 4067-4109.

[22] T. Iwashita, T. Egami, Atomic mechanism of flow in simple liquids under shear Phys. Rev. Lett. 108 (2012) 196001.

[23] B.Y. Cui, J. Yang, J.Q. Qiao, M.Q. Jiang, L.H. Dai, Y.-J. Wang, A. Zaccone, Atomic theory of viscoelastic response and memory effects in metallic glasses, Phys. Rev. B 96 (2017) 094203.

[24] A. Heesemann, V.Zöllmer, K. Rätzke, F. Faupel, Evidence of highly collective Co diffusion in the whole stability range of Co-Zr glasses, Phys. Rev. Lett. 84 (2000) 1467-1470.

[25] V. Zöllmer, K. Rätzke, F. Faupel, A. Rehmet, U. Geyer, Evidence of diffusion via collective hopping in metallic supercooled liquids and glasses, Phys. Rev. B 65 (2002), 220201(R).

[26] M. Leitner, B. Sepiol, L.M. Stadler, B. Pfau, G. Vogl, Atomic diffusion studied with coherent X-rays, Nat. Mater. 8 (2009) 717-720.

[27] Z. Evenson, B. Ruta, S. Hechler, M. Stolpe, E. Pineda, I. Gallino, R. Busch, X-ray photon correlation spectroscopy reveals intermittent aging dynamics in a metallic glass, Phys. Rev. Lett. 115 (2015) 175701.

[28] V.M. Giordano, B. Ruta, Unveiling the structural arrangements responsible for the atomic dynamics in metallic glasses during physical aging, Nat. Commun. 7 (2016) 10344

[29] S. Küchemann, C. Liu, E.M. Dufresne, J. Shin, R. Maaß, Shear banding leads to accelerated aging dynamics in a metallic glass, Phys. Rev. B 97 (2018) 14204

[30] B.A. Pazmiño Betancourt, P.Z. Hanakata, F.W. Starr, J.F. Douglas, Quantitative relations between cooperative motion, emergent elasticity, and free volume in model glass-forming polymer materials, Proc. Natl. Acad. Sci. U. S. A 112 (2015) 2966-2971.

[31] H. Teichler, Mode coupling theory and the glass transition in molecular dynamics simulated Nizr, Phys. Rev. Lett. 76 (1996) 62.

[32] C. Donati, J.F. Douglas, W. Kob, S.J. Plimpton, P.H. Poole, S.C. Glotzer, Stringlike cooperative motion in a supercooled liquid, Phys. Rev. Lett. 80 (1998) 2338-2341.

[33] H. Teichler, Structural dynamics on the us scale in molecular-dynamics simulated, deeply undercooled, glass forming $\mathrm{Ni}_{0.5} \mathrm{Zr}_{0.5}$, J. Non. Cryst. Solids 293-295 (2001) 339-344.

[34] S. Swayamjyoti, J.F. Löffler, P.M. Derlet, Local structural excitations in mode glasses, Phys. Rev. B 89 (2014) 224201.

[35] S. Swayamjyoti, J.F. Löffler, P.M. Derlet, Local structural excitations in mode glass systems under applied load, Phys. Rev. B 93 (2016) 144202.

[36] P.M. Derlet, R. Maaß, Thermal processing and enthalpy storage of a binary amorphous solid: a molecular dynamics study, J. Mater. Res. 32 (2017) 2668-2679.

[37] P.M. Derlet, R. Maaß, Thermally-activated stress relaxation in a model amorphous solid and the formation of a system-spanning shear event, Acta Mater. 143 (2018) 205-213.

[38] D. Rodney, A. Tanguy, D. Vandembroucq, Modeling the mechanics of amorphous solids at different length scale and time scale, Model. Simulat. Mater. Sci. Eng. 19 (2011) 083001.

[39] A. Laio, M. Parrinello, Escaping free-energy minima, Proc. Natl. Acad. Sci. U. S A 99 (2002) 12562-12566.

[40] T.T. Lau, A. Kushima, S. Yip, Atomistic simulation of creep in a nanocrystal, Phys. Rev. Lett. 104 (2010) 175501.

[41] M. Kabir, T.T. Lau, D. Rodney, S. Yip, K.J. Van Vliet, Predicting dislocation climb and creep from explicit atomistic details, Phys. Rev. Lett. 105 (2010) 95501.

[42] Y. Fan, Y. Osetsky, S. Yip, B. Yildiz, Onset mechanism of strain-rate-induced flow stress upturn, Phys. Rev. Lett. 109 (2012) 135503.

[43] J.-P. Du, Y.-J. Wang, Y.-C. Lo, L. Wan, S. Ogata, Mechanism transition and strong temperature dependence of dislocation nucleation from grain boundaries: an accelerated molecular dynamics study, Phys. Rev. B 94 (2016) 104110.

[44] Z. Bai, Y. Fan, Abnormal strain rate sensitivity driven by a unit dislocationobstacle interaction in bcc Fe, Phys. Rev. Lett. 120 (2018) 125504.

[45] A.F. Voter, Hyperdynamics: accelerated molecular dynamics of infrequent events, Phys. Rev. Lett. 78 (1997) 3908-3911.

[46] A. Ishii, S. Ogata, H. Kimizuka, J. Li, Adaptive-boost molecular dynamics simulation of carbon diffusion in iron, Phys. Rev. B 85 (2012) 064303.

[47] L. Sandoval, D. Perez, B.P. Uberuaga, A.F. Voter, Competing kinetics and He bubble morphology in W, Phys. Rev. Lett. 114 (2015) 105502.

[48] L.K. Béland, P. Brommer, F. El-Mellouhi, J.-F. Joly, N. Mousseau, Kinetic activation-relaxation technique, Phys. Rev. E 84 (2011) 46704.

[49] A. Kushima, X. Lin, J. Li, J. Eapen, J.C. Mauro, X.F. Qian, P. Diep, S. Yip,
Computing the viscosity of supercooled liquids, J. Chem. Phys. 130 (2009) 224504.

[50] P.M. Piaggi, O. Valsson, M. Parrinello, Enhancing entropy and enthalpy fluctuations to drive crystallization in atomistic simulations, Phys. Rev. Lett. 119 (2017) 015701.

[51] D. Rodney, C.A. Schuh, Yield stress in metallic glasses: the jammingunjamming transition studied through Monte Carlo simulations based on the activation-relaxation technique, Phys. Rev. B 80 (2009) 184203.

[52] P.H. Cao, X. Lin, H.S. Park, Strain-rate and temperature dependence of yield stress of amorphous solids via self-learning metabasin escape algorithm, J. Mech. Phys. Solid. 68 (2014) 239-250.

[53] P.H. Cao, M.P. Short, S. Yip, Understanding the mechanisms of amorphous creep through molecular simulation, Proc. Natl. Acad. Sci. U. S. A 114 (2017) $13631-13636$.

[54] M.I. Mendelev, M.J. Kramer, R.T. Ott, D.J. Sordelet, Molecular dynamics simulation of diffusion in supercooled Cu-Zr alloys, Philos. Mag. A 89 (2009) 109-126.

[55] Y. Zhang, C.Z. Wang, M.I. Mendelev, F. Zhang, M.J. Kramer, K.M. Ho, Diffusion in a Cu-Zr metallic glass studied by microsecond-scale molecular dynamics simulations, Phys. Rev. B 91 (2015), 180201(R).

[56] A. Barducci, G. Bussi, M. Parrinello, well-tempered metadynamics: a smoothly converging and tunable free-energy method, Phys. Rev. Lett. 100 (2008) 020603.

[57] M. Parrinello, A. Rahman, Polymorphic transitions in single crystals: a new molecular dynamics method, J. Appl. Phys. 52 (1981) 7182.

[58] J.F. Dama, M. Parrinello, G.A. Voth, Well-tempered metadynamics converges asymptotically, Phys. Rev. Lett. 112 (2014) 240602.

[59] E.D. Cubuk, R.J.S. Ivancic, S.S. Schoenholz, D.J. Strickland, A. Basu, Z.S. Davidson, J. Fontaine, J.L. Hor, Y.-R. Huang, Y. Jiang, N.C. Keim, K.D. Koshigan, J.A. Lefever, T. Liu, X.-G. Ma, D.J. Magagnosc, E. Morrow, C.P. Ortiz, J.M. Rieser, A Shavit, T Still, Y Xu, Y Zhang, KN Nordstrom, P.E. Arratia, R.W. Carpick, D.J. Durian, Z. Fakhraai, D.J. Jerolmack, D. Lee, J. Li, R. Riggleman, K.T. Turner, A.G. Yodh, D.S. Gianola, A.J. Liu, Structure-property relationships from universal signatures of plasticity in disordered solids, Science 358 (2017) 1033-1037.

[60] W. Johnson, K. Samwer, A universal criterion for plastic yielding of metallic glasses with a $\left(T / T_{\mathrm{g}}\right)^{2 / 3}$ temperature dependence, Phys. Rev. Lett. 95 (2005) 195501.

[61] Y. Fan, T. Iwashita, T. Egami, How thermally activated deformation starts in metallic glass, Nat. Commun. 5 (2014) 5083.

[62] H. Eyring, The activated complex in chemical reactions, J. Chem. Phys. 3 (1935) 107.

[63] P.G. Debenedetti, F.H. Stillinger, Supercooled liquids and the glass transition, Nature 410 (2001) 259-267.

[64] K. Niss, C. Dalle-Ferrier, B. Frick, D. Russo, J. Dyre, C. Alba-Simionesco, Connection between slow and fast dynamics of molecular liquids around the glass transition, Phys. Rev. E 82 (2010) 021508.

[65] M. Kluge, H.R. Schober, Diffusion and jump-length distribution in liquid and amorphous $\mathrm{Cu}_{33} \mathrm{Zr}_{67}$, Phys. Rev. B 70 (2004), 224209.

[66] P.M. Derlet, R. Maßß, Linking high and low temperature plasticity in bulk metallic glasses: thermal activation, extreme value statistics and kinetic freezing, Philos. Mag. A 93 (2013) 4232-4263.

[67] P. Derlet, R. Maaß, Linking high- and low-temperature plasticity in bulk metallic glasses II: use of a log-normal barrier energy distribution and a meanfield description of high-temperature plasticity, Philos. Mag. A 94 (2014) 2776-2803.

[68] Y. Fan, T. Iwashita, T. Egami, Energy landscape-driven non-equilibrium evolution of inherent structure in disordered material, Nat. Commun. 8 (2017) 15417.

[69] S.K. Sharma, S. Banerjee, A.K. Kuldeep, Jain, Some correlations for diffusion in amorphous alloys, J. Mater. Res. 4 (1989) 603-606.

[70] H. Hahn, R.S. Averback, H.M. Shyu, Diffusion studies in amorphous NiZr alloys and their relevance for solid state amorphizing reactions, J. Less Common Met 140 (1988) 345-352.

[71] E.C. Stelter, D. Lazarus, Diffusion and thermal stability of amorphous copper zirconium, Phys. Rev. B 36 (1987) 9545.

[72] F. Boioli, T. Albaret, D. Rodney, Shear transformation distribution and activation in glasses at the atomic scale, Phys. Rev. E 95 (2017) 033005.

[73] A. Grandjean, P. Blanchard, Y. Limoge, Activation volume for Hf diffusion in an amorphous $\mathrm{Ni}_{0.54} \mathrm{Zr}_{0.46}$ alloy, Phys. Rev. Lett. 78 (1997) 697.

[74] K. Knorr, M.P. Macht, K. Freitag, H. Mehrer, Self-diffusion in the amorphous and supercooled liquid state of the bulk metallic glass $\mathrm{Zr}_{46.75} \mathrm{Ti}_{82}{ }_{25} \mathrm{Cu}_{7.5}$ $\mathrm{Ni}_{10} \mathrm{Be}_{27.5}$, J. Non. Cryst. Solids 250-252 (1999) 669.

[75] P. Klugkist, K. Ratzke, F. Faupel, P.T. Roche, P. Fielitz, V. Naundorf, Structural effects on diffusion in metallic glasses: the pressure dependence of Ni diffusion in as-quenched and relaxed $\mathrm{Co}_{42} \mathrm{Zr}_{58}$, Philos. Mag. Lett. 79 (1999) 827. 